\title{
Nonadditive quantum gravitational interaction for three nonpointlike objects
}

\author{
Yongshun $\mathrm{Hu}^{1,2, *}$ and Hongwei $\mathrm{Yu}^{3, \dagger}$ \\ ${ }^{1}$ School of Fundamental Physics and Mathematical Sciences, \\ Hangzhou Institute for Advanced Study, UCAS, Hangzhou 310024, China \\ ${ }^{2}$ School of Physical Sciences, University of Chinese \\ Academy of Sciences, Beijing 100049, China \\ ${ }^{3}$ Department of Physics and Synergetic Innovation \\ Center for Quantum Effects and Applications, \\ Hunan Normal University, Changsha, Hunan 410081, China
}

\begin{abstract}
We explore the nonadditive three-body quantum gravitational quadrupole interaction among three nonpointlike objects in their ground states in the framework of linearized quantum gravity and find that the interaction exhibits a distance behavior of $r_{A}^{-5} r_{B}^{-5} r_{C}^{-5}$ in the near regime where the interobject distances are small compared with the characteristic transition wavelength of the objects, and $r_{A}^{-5} r_{B}^{-5} r_{C}^{-5}\left(r_{A}+r_{B}+r_{C}\right)^{-1}$ in the far regime where the distances are larger than the characteristic transition wavelength, where $r_{\xi}$ denotes the distance between any two objects except object $\xi(\xi=A, B, C)$. Compared to the additive quantum gravitational interaction between two ground-state objects in vacuum which is always attractive both in the near and far regimes, the nonadditive interaction among three ground-state objects can be either attractive or repulsive depending on the geometrical configuration of these objects. That is, in principle, the attractive or repulsive properties of the nonadditive three-body quantum gravitational interaction can be manipulated by changing the geometrical arrangement of the objects.
\end{abstract}

\footnotetext{
* huyongshun@ucas.ac.cn

† Corresponding author. hwyu@hunnu.edu.cn
} 


\section{INTRODUCTION}

In a quantum sense, there inevitably exist quantum fluctuations of fields in vacuum, which may induce observable effects. In general, fluctuating fields in vacuum induce instantaneous multipole moments in atoms or objects, which then interact via the exchange of virtual mediating particles, and an interaction energy is thus resulted. One of the wellknow examples is the electromagnetic Casimir-Polder (CP) interaction, which arises from the vacuum-fluctuation-induced dipole-dipole interaction between two neutral atoms via the exchange of virtual photons [1]. Such interatomic CP effects have been widely investigated in various circumstances, e.g., in the presence of external electromagnetic fields [2-12], thermal fluctuations [13, 14], and boundaries [15, 16]. Also, it has been found that the interatomic $\mathrm{CP}$ interactions behave differently in terms of distance-dependence when the two atoms are in different quantum states [17-30].

Likewise, in the gravitational case, there should also exist vacuum-fluctuation-induced quantum gravitational quadrupole-quadrupole interactions between nonpointlike objects, if one accepts that basic quantum principles are applicable to gravity as well. However, a full theory of quantum gravity has not yet been established. Nonetheless, one can still investigate low energy quantum gravitational effects in the framework of the effective field theory or linearized quantum gravity. Such investigations are based on the belief that the results obtained should agree with those in a full theory of quantum gravity at low energy scales. Similar to the electromagnetic case, the gravitational CP-like quadrupole-quadrupole interactions are found to be dependent on the quantum states of the two-object systems [31-35] and can be modified by the thermal fluctuations of gravitons [33], the gravitational boundaries [36], and the external gravitational radiation fields [37, 38]. For example, the vacuum-fluctuation-induced gravitational quadrupole-quadrupole potential for two groundstate nonpointlike objects behaves as $r^{-10}$ and $r^{-11}$ in the near and far regimes respectively, while for two entangled objects it behaves as $r^{-5}$ and $r^{-1}$ in the near and far regimes

respectively. The presence of gravitational boundaries or a thermal bath of gravitons causes temperature-dependent or boundary-dependent modifications to the interaction potentials in vacuum [33, 36]. Moreover, in the presence of external gravitational radiation fields, the induced interobject quantum gravitational quadrupole-quadrupole interaction can be attractive or repulsive depending on the properties (e.g., polarization et al.) of the external 
gravitational fields $[37,38]$, which is significantly different from that of the vacuum case which is always attractive.

The CP-like quantum gravitational interactions discussed above are obviously additive, since the corresponding interaction potentials are related just to the distance between the pair. Naturally, a question arises as to whether the CP-like quantum gravitational interaction is still additive in the presence of a third nonpointlike object, or in other words, whether a nonadditive three-body quantum gravitational quadrupole interaction exists. Again, there are similar examples in quantum electrodynamics. It has been demonstrated that the electromagnetic CP interaction between two neutral atoms can be modified by the presence of a third one which then generates a triple-dipole interaction potential and gives rise to a nonadditive three-atom interaction [39-44]. Physically speaking, the electromagnetic three-body interaction among three neutral atoms in vacuum arises from the processes of three-photon exchange wherein only one photon is exchanged between each pair of atoms, so that the interaction potential is related to distances between every pair of atoms and is thus nonadditive. Such correlated interaction processes among three atoms are clearly different from those of the two-atom case, which arise from two-photon exchange between the pair and from which the resulting interaction is obviously additive. Similarly, in the gravitational case, one may also expect a nonadditive three-body quantum gravitational quadrupole interaction when a third nonpointlike object is present.

In this paper, we explore the nonadditive three-body quantum gravitational quadrupole interaction among three nonpointlike objects in their ground states based on the theory of linearized quantum gravity. First, we derive the three-body quantum gravitational potential in the framework of linearized quantum gravity. Then, we discuss its behaviors in specific cases and show concretely a geometrical dependence of the attractive or repulsive properties of the interaction. Throughout this paper, the Latin indices and the Greek indices run from 1 to 3 and 0 to 3, respectively. The Einstein summation convention for repeated indices is assumed and units with $\hbar=c=16 \pi G=1$ are used, where $\hbar$ is the reduced Planck constant, $c$ is the speed of light and $G$ is the Newtonian gravitational constant. 


\section{BASIC EQUATIONS}

We consider three nonpointlike objects (labeled as A, B and C) coupled with the fluctuating gravitational fields in vacuum. For simplicity, let us model these objects by two-level systems with the ground and excited states being $\left|g_{\xi}\right\rangle$ and $\left|e_{\xi}\right\rangle$ respectively, and label the corresponding energy spacing as $\omega_{\xi}(\xi=A, B, C)$. The total Hamiltonian of the system can be written as

$$
H=H_{F}+H_{S}+H_{I}
$$

where $H_{F}$ is the Hamiltonian of the gravitational fields, $H_{S}$ represents the Hamiltonian of the three nonpointlike objects $\left(\mathrm{A}, \mathrm{B}\right.$ and $\mathrm{C}$ ), and $H_{I}$ denotes the interaction Hamiltonian between the nonpointlike objects and the gravitational fields, which takes the form

$$
H_{I}=-\frac{1}{2} Q_{i j}^{A} E_{i j}\left(\vec{x}_{A}\right)-\frac{1}{2} Q_{i j}^{B} E_{i j}\left(\vec{x}_{B}\right)-\frac{1}{2} Q_{i j}^{C} E_{i j}\left(\vec{x}_{C}\right)
$$

where $Q_{i j}^{\xi}$ is the quadrupole moment operator of object $\xi(\xi=A, B, C)$, and $E_{i j}$ is the gravitoelectric tensor of the fluctuating gravitational fields in vacuum, which is defined as $E_{i j}=C_{0 i 0 j}$ by an analogy between the linearized Einstein field equations and the Maxwell equations [45-51], where $C_{\mu \nu \alpha \beta}$ is the Weyl tensor. Under the weak-field approximation, the spacetime metric can be written as a sum of the flat spacetime metric and a linearized perturbation $h_{\mu \nu}$. Then, the gravitoelectric tensor $E_{i j}$ is found to be

$$
E_{i j}=\frac{1}{2} \ddot{h}_{i j}
$$

In the transverse traceless gauge, the linearized metric perturbation can be quantized as [52]

$$
h_{i j}=\sum_{\vec{p}, \lambda} \sqrt{\frac{1}{2 \omega(2 \pi)^{3}}}\left[a_{\lambda}(\omega) e_{i j}^{(\lambda)} e^{i(\vec{p} \cdot \vec{x}-\omega t)}+\text { H.c. }\right] \text {, }
$$

where $a_{\lambda}(\omega)$ is the annihilation operator of the fluctuating gravitational fields, $e_{i j}^{(\lambda)}$ are polarization tensors, $\lambda$ labels the polarization states, $\omega=|\vec{p}|=\left(p_{x}^{2}+p_{y}^{2}+p_{z}^{2}\right)^{1 / 2}$, and H.c. denotes the Hermitian conjugate.

We choose the initial state of the whole system as

$$
|\phi\rangle=\left|g_{A}\right\rangle\left|g_{B}\right\rangle\left|g_{C}\right\rangle|0\rangle
$$

and denote its energy as $E_{\phi}$, where $|0\rangle$ represents the vacuum state of the fluctuating gravitational field. In analogy to the electromagnetic case [40], the nonadditive three-body 
quantum gravitational interaction energy can be obtained from the sixth-order perturbation theory, which contains 360 possible time-ordered diagrams, and a typical one is shown in Fig. 1. However, a direct sixth-order calculation is exceedingly unwieldy and, for conve-

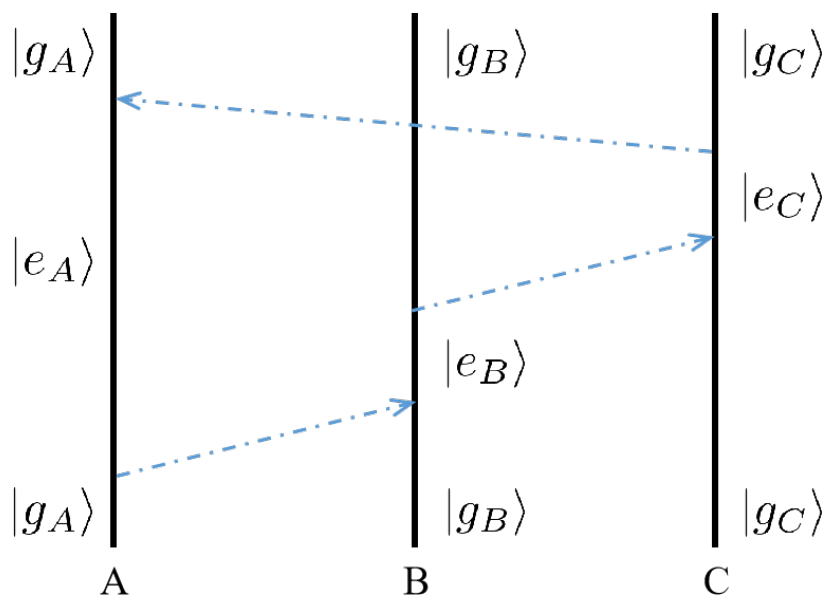

FIG. 1. One typical time-ordered diagram for the calculation of the nonadditive three-body quantum gravitational interaction. The blue dotted line represents a virtual graviton.

nience, simplifications of the interaction processes are needed. For this purpose, an effective two-graviton interaction Hamiltonian is introduced, and for the first step, we re-express the vacuum-fluctuation-induced quadrupole in object $\xi$ as

$$
Q_{i j}^{\xi}=\frac{1}{2} \alpha_{i j k l}^{\xi}(\omega) E_{k l}
$$

where $\alpha_{i j k l}^{\xi}(\omega)$ is the gravitational polarizability of object $\xi$, which takes the form

$$
\alpha_{i j k l}^{\xi}(\omega)=\frac{2 \omega_{\xi} \hat{Q}_{i j}^{\xi} \hat{Q}_{k l}^{\xi}}{\omega_{\xi}^{2}-\omega^{2}}
$$

where $\hat{Q}_{i j}^{\xi}$ represents the quadrupole transition moments. Then, the effective Hamiltonian can be written as

$$
H_{I}^{e f f}=-\frac{1}{4} \sum_{\xi=A, B, C} \alpha_{i j k l}^{\xi}(\omega) E_{i j}\left(\vec{x}_{\xi}\right) E_{k l}\left(\vec{x}_{\xi}\right) .
$$

The three-body quantum gravitational interaction energy shift can be calculated based on the third-order perturbation theory

$$
\Delta E=\sum_{I, I I} \frac{\left\langle\phi\left|H_{I}^{e f f}\right| I I\right\rangle\left\langle I I\left|H_{I}^{e f f}\right| I\right\rangle\left\langle I\left|H_{I}^{e f f}\right| \phi\right\rangle}{\left(E_{I I}-E_{\phi}\right)\left(E_{I}-E_{\phi}\right)}
$$




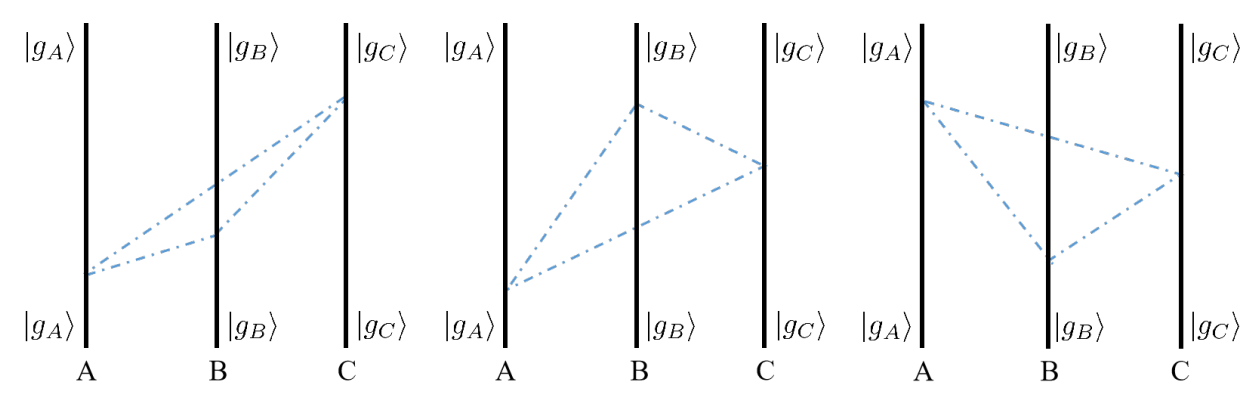

(1)

(2)

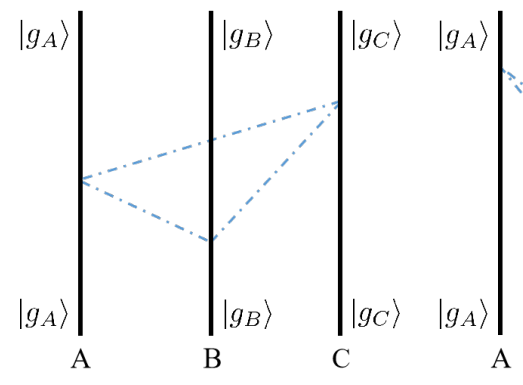

(4)

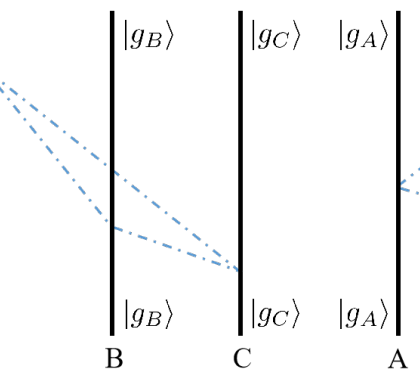

(5)
(3)

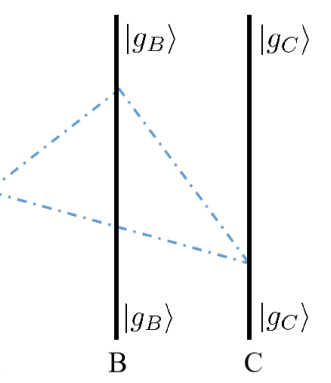

(6)

FIG. 2. Time-ordered diagrams contributing to three-body quantum gravitational interaction when an effective two-graviton coupling Hamiltonian is employed.

with only six contributing time-ordered diagrams as shown in Fig. 2. Summing up all the contributions, the three-body gravitational interaction is found to be

$$
\begin{aligned}
\Delta E_{A B C}= & -\frac{1}{2^{15}(2 \pi)^{9}} \sum_{\vec{p}_{1}, \lambda_{1}} \sum_{\vec{p}_{2}, \lambda_{2}} \sum_{\vec{p}_{3}, \lambda_{3}}\left[\alpha_{i j k l}^{A}\left(\omega_{1}\right)+\alpha_{i j k l}^{A}\left(\omega_{3}\right)\right]\left[\alpha_{g h m n}^{B}\left(\omega_{1}\right)+\alpha_{g h m n}^{B}\left(\omega_{2}\right)\right] \\
& \times\left[\alpha_{e f q s}^{C}\left(\omega_{2}\right)+\alpha_{e f q s}^{C}\left(\omega_{3}\right)\right] e_{i j}^{\left(\lambda_{1}\right)} e_{k l}^{\left(\lambda_{3}\right)} e_{g h}^{\left(\lambda_{1}\right)} e_{m n}^{\left(\lambda_{2}\right)} e_{e f}^{\left(\lambda_{2}\right)} e_{q s}^{\left(\lambda_{3}\right)} e^{i \vec{p}_{1} \cdot \vec{r}_{C}} e^{i \vec{p}_{2} \cdot \vec{r}_{A}} e^{i \vec{p}_{3} \cdot \vec{r}_{B}} \\
& \times\left[\frac{2}{\left(\omega_{1}+\omega_{3}\right)\left(\omega_{1}+\omega_{2}\right)}+\frac{2}{\left(\omega_{1}+\omega_{2}\right)\left(\omega_{2}+\omega_{3}\right)}+\frac{2}{\left(\omega_{1}+\omega_{3}\right)\left(\omega_{2}+\omega_{3}\right)}\right],
\end{aligned}
$$

where $\vec{r}_{A}=\vec{x}_{C}-\vec{x}_{B}, \vec{r}_{B}=\vec{x}_{A}-\vec{x}_{C}$, and $\vec{r}_{C}=\vec{x}_{B}-\vec{x}_{A}$ have been defined. Using the summation of polarization tensors in the transverse traceless gauge [52]

$$
\begin{aligned}
\sum_{\lambda} e_{i j}^{(\lambda)} e_{k l}^{(\lambda)}= & \delta_{i k} \delta_{j l}+\delta_{i l} \delta_{j k}-\delta_{i j} \delta_{k l}+\hat{p}_{i} \hat{p}_{j} \delta_{k l}+\hat{p}_{k} \hat{p}_{l} \delta_{i j} \\
& -\hat{p}_{i} \hat{p}_{k} \delta_{j l}-\hat{p}_{i} \hat{p}_{l} \delta_{j k}-\hat{p}_{j} \hat{p}_{k} \delta_{i l}-\hat{p}_{j} \hat{p}_{l} \delta_{i k}+\hat{p}_{i} \hat{p}_{j} \hat{p}_{k} \hat{p}_{l}
\end{aligned}
$$

where $\hat{p}_{i}$ is the $i$-th component of the unit vector $\vec{p} / p$, we obtain

$$
\begin{aligned}
\sum_{\lambda} e_{i j}^{(\lambda)} e_{k l}^{(\lambda)} e^{i \vec{p} \cdot \vec{r}}= & \frac{1}{\omega^{4}}\left[\left(\delta_{i k} \delta_{j l}+\delta_{i l} \delta_{j k}-\delta_{i j} \delta_{k l}\right) \nabla^{4}+\left(\partial_{i} \partial_{j} \delta_{k l}+\partial_{k} \partial_{l} \delta_{i j}\right.\right. \\
& \left.\left.-\partial_{i} \partial_{k} \delta_{j l}-\partial_{i} \partial_{l} \delta_{j k}-\partial_{j} \partial_{k} \delta_{i l}-\partial_{j} \partial_{l} \delta_{i k}\right) \nabla^{2}+\partial_{i} \partial_{j} \partial_{k} \partial_{l}\right] e^{i \vec{p} \cdot \vec{r}} \\
= & \frac{1}{\omega^{4}} \hat{H}_{i j k l}^{r} e^{i \vec{p} \cdot \vec{r}}
\end{aligned}
$$


and

$$
\int \frac{1}{\omega^{4}} \hat{H}_{i j k l}^{r} e^{i \vec{p} \cdot \vec{r}} d \Omega_{\vec{p}}=4 \pi \hat{H}_{i j k l}^{r} \frac{\sin \omega r}{\omega^{5} r}
$$

where $\hat{H}_{i j k l}^{r}$ is a differential operator whose definition follows directly from Eq. (12), $\nabla^{2}=$ $\partial_{i} \partial^{i}$, and $\Omega_{\vec{p}}$ denotes the solid angle. Replacing the summation over wave vectors and polarization states in Eq. (10) by integration and performing the integral with the help of the integral representation

$$
\begin{aligned}
& \frac{1}{\left(\omega_{1}+\omega_{3}\right)\left(\omega_{1}+\omega_{2}\right)}+\frac{1}{\left(\omega_{1}+\omega_{2}\right)\left(\omega_{2}+\omega_{3}\right)}+\frac{1}{\left(\omega_{1}+\omega_{3}\right)\left(\omega_{2}+\omega_{3}\right)} \\
& =\frac{4}{\pi} \int_{0}^{\infty} \frac{\omega_{1} \omega_{2} \omega_{3} d u}{\left(\omega_{1}^{2}+u^{2}\right)\left(\omega_{2}^{2}+u^{2}\right)\left(\omega_{3}^{2}+u^{2}\right)}
\end{aligned}
$$

we obtain

$$
\begin{aligned}
\Delta E_{A B C}= & -\frac{1}{2^{9}(2 \pi)^{6}} \hat{H}_{m n e f}^{r_{A}} \hat{H}_{k l q s}^{r_{B}} \hat{H}_{i j g h}^{r_{C}} \int_{0}^{\infty} d \omega_{1} \int_{0}^{\infty} d \omega_{2} \int_{0}^{\infty} d \omega_{3}\left[\alpha_{i j k l}^{A}\left(\omega_{1}\right)+\alpha_{i j k l}^{A}\left(\omega_{3}\right)\right] \\
& \times\left[\alpha_{g h m n}^{B}\left(\omega_{1}\right)+\alpha_{g h m n}^{B}\left(\omega_{2}\right)\right]\left[\alpha_{e f q s}^{C}\left(\omega_{2}\right)+\alpha_{e f q s}^{C}\left(\omega_{3}\right)\right] \frac{\sin \omega_{2} r_{A} \sin \omega_{3} r_{B} \sin \omega_{1} r_{C}}{\pi r_{A} r_{B} r_{C}} \\
& \times \int_{0}^{\infty} \frac{\omega_{1} \omega_{2} \omega_{3} d u}{\left(\omega_{1}^{2}+u^{2}\right)\left(\omega_{2}^{2}+u^{2}\right)\left(\omega_{3}^{2}+u^{2}\right)} .
\end{aligned}
$$

To evaluate the frequency integrals in Eq. (15), an integral representation is utilized, i.e.,

$$
\int_{0}^{\infty} \alpha_{i j k l}^{\xi}(p) \frac{p \sin p r}{p^{2}+u^{2}} d p=\frac{1}{4 i} \int_{-\infty}^{\infty} \alpha_{i j k l}^{\xi}(p) e^{i p r}\left(\frac{1}{p+i u}+\frac{1}{p-i u}\right) d p=\frac{\pi}{2} \alpha_{i j k l}^{\xi}(i u) e^{-u r}
$$

Then, the expression of the nonadditive three-body quantum gravitational quadrupole interaction is finally obtained

$$
\Delta E_{A B C}=-\frac{1}{2^{15} \pi^{4}} \hat{H}_{m n e f}^{r_{A}} \hat{H}_{k l q s}^{r_{B}} \hat{H}_{i j g h}^{r_{C}} \int_{0}^{\infty} \alpha_{i j k l}^{A}(i u) \alpha_{g h m n}^{B}(i u) \alpha_{e f q s}^{C}(i u) \frac{e^{-u\left(r_{A}+r_{B}+r_{C}\right)}}{r_{A} r_{B} r_{C}} d u
$$

Now discussions about the three-body interaction potential Eq. (17) are in order. For simplicity, we assume that the objects $\mathrm{A}, \mathrm{B}$, and $\mathrm{C}$ are isotropically polarizable so that

$$
\alpha_{i j k l}^{\xi}(\omega)=\left(\delta_{i k} \delta_{j l}+\delta_{i l} \delta_{j k}\right) \alpha^{\xi}(\omega)
$$

where $\alpha^{\xi}(\omega)$ denotes the isotropic polarizability of object $\xi$. Then, Eq. (17) can be simplified as

$$
\Delta E_{A B C}=-\frac{1}{2^{12} \pi^{4}} \hat{H}_{k l m n}^{r_{A}} \hat{H}_{i j m n}^{r_{B}} \hat{H}_{i j k l}^{r_{C}} \int_{0}^{\infty} \alpha^{A}(i u) \alpha^{B}(i u) \alpha^{C}(i u) \frac{e^{-u\left(r_{A}+r_{B}+r_{C}\right)}}{r_{A} r_{B} r_{C}} d u .
$$

First, in the near regime, where the interobject distance $r_{A}, r_{B}$, and $r_{C}$ are all small compared with the characteristic transition wavelength of the objects, the integral in Eq. (19) is 
effectively limited to the region where $e^{-u\left(r_{A}+r_{B}+r_{C}\right)} \approx 1$ and the asymptotic result is then obtained as

$$
\Delta E_{A B C} \simeq-\frac{1}{2^{12} \pi^{4}} \hat{H}_{k l m n}^{r_{A}} \hat{H}_{i j m n}^{r_{B}} \hat{H}_{i j k l}^{r_{C}} \frac{1}{r_{A} r_{B} r_{C}} \int_{0}^{\infty} \alpha^{A}(i u) \alpha^{B}(i u) \alpha^{C}(i u) d u .
$$

Notice that

$$
\begin{aligned}
\hat{H}_{k l m n}^{r} \frac{1}{r}= & \frac{1}{r^{5}}\left[3\left(\delta_{k l} \delta_{m n}+\delta_{k m} \delta_{l n}+\delta_{k n} \delta_{l m}\right)-15\left(\hat{r}_{k} \hat{r}_{l} \delta_{m n}+\hat{r}_{m} \hat{r}_{n} \delta_{k l}\right.\right. \\
& \left.\left.+\hat{r}_{k} \hat{r}_{m} \delta_{l n}+\hat{r}_{k} \hat{r}_{n} \delta_{l m}+\hat{r}_{l} \hat{r}_{m} \delta_{k n}+\hat{r}_{l} \hat{r}_{n} \delta_{k m}\right)+105 \hat{r}_{k} \hat{r}_{l} \hat{r}_{m} \hat{r}_{n}\right]
\end{aligned}
$$

where $\hat{r}_{n}$ is the $n$-th component of the unit vector $\vec{r} / r\left(r=r_{A}, r_{B}, r_{C}\right)$. After some algebraic manipulations, we obtain the near-zone three-body quantum gravitational interaction potential

$$
\Delta E_{A B C} \simeq-\frac{405}{2^{12} \pi^{4} r_{A}^{5} r_{B}^{5} r_{C}^{5}} F\left(\theta_{A}, \theta_{B}, \theta_{C}\right) \int_{0}^{\infty} \alpha^{A}(i u) \alpha^{B}(i u) \alpha^{C}(i u) d u
$$

with

$$
\begin{aligned}
F\left(\theta_{A}, \theta_{B}, \theta_{C}\right)= & 18+110 \cos \theta_{A} \cos \theta_{B} \cos \theta_{C}-35\left(\cos ^{2} \theta_{A} \cos ^{2} \theta_{C}+\cos ^{2} \theta_{B} \cos ^{2} \theta_{C}\right. \\
& \left.+\cos ^{2} \theta_{A} \cos ^{2} \theta_{B}\right)+245 \cos ^{2} \theta_{A} \cos ^{2} \theta_{B} \cos ^{2} \theta_{C},
\end{aligned}
$$

where $\theta_{A}, \theta_{B}$, and $\theta_{C}$ are the internal angles of the triangle which are opposite to sides $B C, C A$, and $A B$, respectively. A schematic diagram of the geometrical configuration for the three objects is shown in Fig. 3. Here $\cos \theta_{A}=-\hat{r}_{B} \cdot \hat{r}_{C}, \cos \theta_{B}=-\hat{r}_{A} \cdot \hat{r}_{C}$ and

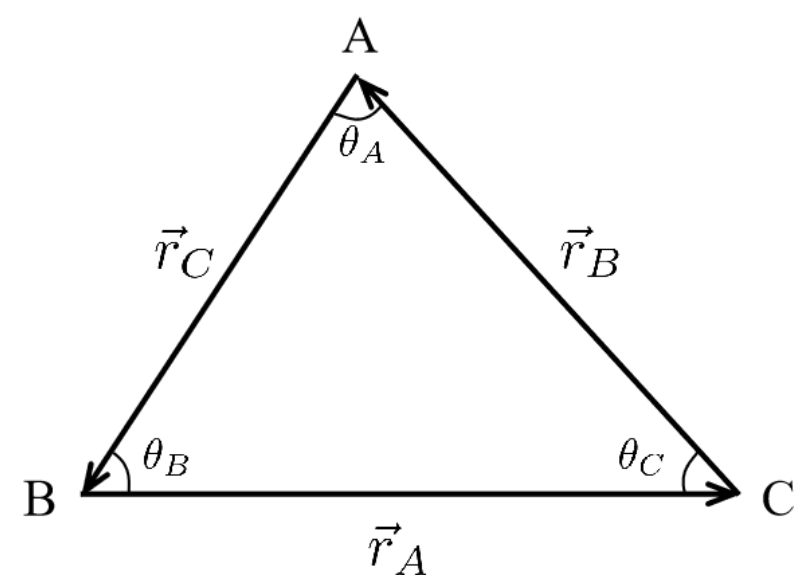

FIG. 3. The notations for the vertices and interobject distance vector used for three objects.

$\cos \theta_{C}=-\hat{r}_{A} \cdot \hat{r}_{B}$ are used, and the identity for internal angles of a triangle, i.e.,

$$
\cos ^{2} \theta_{A}+\cos ^{2} \theta_{B}+\cos ^{2} \theta_{C}=1-2 \cos \theta_{A} \cos \theta_{B} \cos \theta_{C}
$$


has been applied. Notice that, the function $F\left(\theta_{A}, \theta_{B}, \theta_{C}\right)$ can be either positive or negative depending on the geometrical configuration, and as a result the interaction can be attractive or repulsive. Several concrete examples are helpful to illustrate such a geometrical dependence or influence. For a linear arrangement of the three objects with $r_{A}=2 r_{B}=2 r_{C}=2 r$, $\theta_{A}=\pi$, and $\theta_{B}=\theta_{C}=0$, the three-body interaction potential Eq. (22) is

$$
\Delta E_{A B C} \simeq-\frac{1215}{2^{13} \pi^{4} r^{15}} \int_{0}^{\infty} \alpha^{A}(i u) \alpha^{B}(i u) \alpha^{C}(i u) d u
$$

So, the three-body quantum gravitational quadrupole interaction exhibits an $r^{-15}$ dependence in the near regime. Since the sign of the potential Eq. (25) is negative, the interaction is attractive for the case of a linear geometrical arrangement. For non-linear arrangements of the three objects, we now consider special two cases, which give rise to a positive and a negative value of $F\left(\theta_{A}, \theta_{B}, \theta_{C}\right)$ respectively. For an equilateral triangle in which $r_{A}=r_{B}=r_{C}=r$ with $\theta_{A}=\theta_{B}=\theta_{C}=\frac{\pi}{3}$, the interaction potential Eq. (22) is

$$
\Delta E_{A B C} \simeq-\frac{752085}{2^{18} \pi^{4} r^{15}} \int_{0}^{\infty} \alpha^{A}(i u) \alpha^{B}(i u) \alpha^{C}(i u) d u,
$$

while for a triangle in which $r_{A}=\sqrt{3} r_{B}=\sqrt{3} r_{C}=\sqrt{3} r$ with $\theta_{A}=\frac{2}{3} \pi, \theta_{B}=\theta_{C}=\frac{\pi}{6}$, the potential Eq. (22) is

$$
\Delta E_{A B C} \simeq \frac{20745 \sqrt{3}}{2^{18} \pi^{4} r^{15}} \int_{0}^{\infty} \alpha^{A}(i u) \alpha^{B}(i u) \alpha^{C}(i u) d u .
$$

Obviously, the distance dependence of such a three-body interaction in the triangle configurations is also $r^{-15}$. Notice that the sign of Eq. (26) is negative while that of Eq. (27) is positive, which respectively implies an attractive and a repulsive force associated with the corresponding geometrical arrangement of the three objects. That is, in the near regime, the attractive or repulsive properties of the three-body quantum gravitational potential depend crucially on the geometrical configuration of the three objects.

Second, in the far regime, where the interobject distance $r_{A}, r_{B}$, and $r_{C}$ are all larger than the characteristic transition wavelength of the objects so that the polarizability $\alpha^{\xi}(i u)$ can be approximately replaced by the static one (i.e., $\alpha^{\xi}(0)$ ), since, in the present case, small values of $u$ could provide the dominating contribution due to the existence of an exponential in the integrand in Eq. (19), we obtain

$$
\begin{aligned}
\Delta E_{A B C} & \simeq-\frac{1}{2^{12} \pi^{4}} \alpha^{A}(0) \alpha^{B}(0) \alpha^{C}(0) \hat{H}_{k l m n}^{r_{A}} \hat{H}_{i j m n}^{r_{B}} \hat{H}_{i j k l}^{r_{C}} \int_{0}^{\infty} \frac{e^{-u\left(r_{A}+r_{B}+r_{C}\right)}}{r_{A} r_{B} r_{C}} d u \\
& =-\frac{1}{2^{12} \pi^{4}} \alpha^{A}(0) \alpha^{B}(0) \alpha^{C}(0) \hat{H}_{k l m n}^{r_{A}} \hat{H}_{i j m n}^{r_{B}} \hat{H}_{i j k l}^{r_{C}} \frac{1}{r_{A} r_{B} r_{C}\left(r_{A}+r_{B}+r_{C}\right)} .
\end{aligned}
$$


Note that a detailed calculation of the derivative in Eq. (28) is complicated and tedious, and it is also profitless to take up much space to show such an expression. For simplicity, here we only give several special examples with certain geometrical configurations of the three objects, which may be enough to show the essential properties of the three-body quantum gravitational potential in the far regime. Also, a serviceable derivative may be helpful, which is given as follows

$$
\begin{aligned}
\hat{H}_{k l m n}^{r} \frac{e^{-u r}}{r}= & \frac{e^{-u r}}{r^{5}}\left[U_{1}(u r)\left(\delta_{k m} \delta_{l n}+\delta_{k n} \delta_{l m}\right)+U_{2}(u r) \delta_{k l} \delta_{m n}+U_{3}(u r)\left(\hat{r}_{k} \hat{r}_{l} \delta_{m n}+\hat{r}_{m} \hat{r}_{n} \delta_{k l}\right)\right. \\
& \left.+U_{4}(u r)\left(\hat{r}_{k} \hat{r}_{m} \delta_{l n}+\hat{r}_{k} \hat{r}_{n} \delta_{l m}+\hat{r}_{l} \hat{r}_{m} \delta_{k n}+\hat{r}_{l} \hat{r}_{n} \delta_{k m}\right)+U_{5}(u r) \hat{r}_{k} \hat{r}_{l} \hat{r}_{m} \hat{r}_{n}\right]
\end{aligned}
$$

with

$$
\begin{aligned}
& U_{1}(x)=x^{4}+2 x^{3}+3 x^{2}+3 x+3, \\
& U_{2}(x)=-x^{4}-2 x^{3}-x^{2}+3 x+3, \\
& U_{3}(x)=x^{4}+2 x^{3}-3 x^{2}-15 x-15, \\
& U_{4}(x)=-x^{4}-4 x^{3}-9 x^{2}-15 x-15, \\
& U_{5}(x)=x^{4}+10 x^{3}+45 x^{2}+105 x+105 .
\end{aligned}
$$

For a linear arrangement with $r_{A}=2 r_{B}=2 r_{C}=2 r$, the far-zone three-body interaction potential Eq. (28) becomes

$$
\Delta E_{A B C} \approx-\frac{0.1}{\pi^{4} r^{16}} \alpha^{A}(0) \alpha^{B}(0) \alpha^{C}(0)
$$

Obviously, the three-body quantum gravitational quadrupole interaction shows an $r^{-16}$ dependence in the far regime and is attractive since the sign of the potential Eq. (35) is negative. For the triangle configurations, we also consider two examples with special internal angles to show the geometrical dependence of the attractive or repulsive properties of the far-zone three-body interaction. For an equilateral triangle in which $r_{A}=r_{B}=r_{C}=r$, the far-zone interaction potential Eq. (28) becomes

$$
\Delta E_{A B C} \approx-\frac{7.5}{\pi^{4} r^{16}} \alpha^{A}(0) \alpha^{B}(0) \alpha^{C}(0)
$$

whereas for a triangle in which $r_{A}=r$ with $\theta_{A}=\frac{2}{9} \pi, \theta_{B}=\frac{\pi}{9}, \theta_{C}=\frac{2 \pi}{3}$, the far-zone potential Eq. (28) is

$$
\Delta E_{A B C} \approx \frac{2.4}{\pi^{4} r^{16}} \alpha^{A}(0) \alpha^{B}(0) \alpha^{C}(0)
$$


where the relation $\frac{r_{A}}{\sin \theta_{A}}=\frac{r_{B}}{\sin \theta_{B}}=\frac{r_{C}}{\sin \theta_{C}}$ has been utilized. Obviously, the distance dependence of the far-zone three-body quantum gravitational interaction in the triangle configurations is still $r^{-16}$. Moreover, the sign of Eq. (36) is negative so that the corresponding force is attractive, while that of Eq. (37) is positive and therefore the force is repulsive. Thus, in the far regime, the three-body quantum gravitational quadrupole interaction can also be attractive or repulsive depending on the geometrical configuration of the three objects, which is similar to the case in the near regime.

\section{DISCUSSION}

In this paper, we study the nonadditive three-body quantum gravitational quadrupole interaction between three ground-state nonpointlike objects coupled with the fluctuating gravitational fields in vacuum, based on the perturbation theory in the framework of linearized quantum gravity. Our result shows that the three-body quantum gravitational interaction displays a distance behavior of $r_{A}^{-5} r_{B}^{-5} r_{C}^{-5}$ in the near regime where the interobject distances are smaller than the characteristic transition wavelength of the objects, and $r_{A}^{-5} r_{B}^{-5} r_{C}^{-5}\left(r_{A}+r_{B}+r_{C}\right)^{-1}$ in the far regime where the distances are larger than the characteristic transition wavelength. Here $r_{\xi}$ denotes the distance between any two objects except object $\xi(\xi=A, B, C)$. We also find that, both in the near and far regimes, such a three-body interaction is attractive for a linear arrangement and can be attractive or repulsive depending on the internal angles for a triangle configuration. That is, the attractive or repulsive properties of the nonadditive three-body quantum gravitational quadrupole interaction can in principle be manipulated by changing the geometrical configuration of the three objects, in contrast to the additive two-body case which is always attractive. 


\section{ACKNOWLEDGMENTS}

We would like to thank Jiawei Hu for helpful discussions. This work was supported in part by the NSFC under Grants No. 11690034 and No. 12075084.

[1] H. B. G. Casimir and D. Polder, The influence of retardation on the London-van der Waals forces, Phys. Rev. 73, 360 (1948).

[2] T. Thirunamachandran, Intermolecular interactions in the presence of an intense radiation field, Mol. Phys. 40, 393 (1980).

[3] P. W. Milonni and M.-L. Shih, Source theory of the Casimir force, Phys. Rev. A 45, 4241 (1992).

[4] P. W. Milonni and A. Smith, van der Waals dispersion forces in electromagnetic fields, Phys. Rev. A 53, 3484 (1996).

[5] D. S. Bradshaw and D. L. Andrews, Optically induced forces and torques: Interactions between nanoparticles in a laser beam, Phys. Rev. A 72, 033816 (2005).

[6] D. L. Andrews, R. G. Crisp, and D. S. Bradshaw, Optically induced inter-particle forces: From the bonding of dimers to optical electrostriction in molecular solids, J. Phys. B 39, S637 (2006).

[7] A. Salam, Intermolecular interactions in a radiation field via the method of induced moments, Phys. Rev. A 73, 013406 (2006).

[8] A. Salam, Two alternative derivations of the static contribution to the radiation-induced intermolecular energy shift, Phys. Rev. A 76, 063402 (2007).

[9] Y. Sherkunov, Casimir-Polder interaction between two atoms in electromagnetic fields, J. Phys. Conf. Ser. 161, 012041 (2009).

[10] W. C. Mackrodt, The coupling of intermolecular interactions to a static electric field, Mol. Phys. 27, 933 (1974).

[11] G. Fiscelli, L. Rizzuto, and R. Passante, Dispersion Interaction between Two Hydrogen Atoms in a Static Electric Field, Phys. Rev. Lett. 124, 013604 (2020).

[12] Y. Hu, J. Hu, and H. Yu, Quantum corrections to the classical electrostatic interaction between induced dipoles, Phys. Rev. A 103, 042803 (2021). 
[13] G. H. Goedecke and Roy C. Wood, Casimir-Polder interaction at finite temperature, Phys. Rev. A 60, 2577 (1999).

[14] H. Wennerström, J. Daicic, and B. W. Ninham, Temperature dependence of atom-atom interactions, Phys. Rev. A 60, 25811999.

[15] E. A. Power and T. Thirunamachandran, Quantum electrodynamics in a cavity, Phys. Rev. A 25, 2473 (1982).

[16] S. Spagnolo, R. Passante, and L. Rizzuto, Field fluctuations near a conducting plate and Casimir-Polder forces in the presence of boundary conditions, Phys. Rev. A 73, 062117 (2006).

[17] R. R. McLone and E. A. Power, The long range van der Waals forces between non-identical systems, Proc. R. Soc. London A 286, 573 (1965).

[18] L. Gomberoff, R. R. McLone, and E. A. Power, Long-Range Retarded Potentials between Molecules, J. Chem. Phys. 44, 4148 (1966).

[19] E. A. Power and T. Thirunamachandran, Quantum electrodynamics with nonrelativistic sources. V. Electromagnetic field correlations and intermolecular interactions between molecules in either ground or excited states, Phys. Rev. A 47, 2539 (1993).

[20] E. A. Power and T. Thirunamachandran, A new insight into the mechanism of intermolecular forces, Chem. Phys. 171, 1 (1993).

[21] E. A. Power and T. Thirunamachandran, Dispersion forces between molecules with one or both molecules excited, Phys. Rev. A 51, 3660 (1995).

[22] L. Rizzuto, R. Passante, and F. Persico, Dynamical Casimir-Polder energy between an excitedand a ground-state atom, Phys. Rev. A 70, 012107 (2004).

[23] Y. Sherkunov, Casimir-Polder interaction between an excited atom and a gas dielectric medium, Phys. Rev. A 75, 012705 (2007).

[24] J. Preto and M. Pettini, Resonant long-range interactions between polar macromolecules, Phys. Lett. A 377, 587 (2013).

[25] M. Donaire, R. Guérout, and A. Lambrecht, Quasiresonant van der Waals Interaction between Nonidentical Atoms, Phys. Rev. Lett. 115, 033201 (2015).

[26] P. W. Milonni and S. M. H. Rafsanjani, Distance dependence of two-atom dipole interactions with one atom in an excited state, Phys. Rev. A 92, 062711 (2015).

[27] P. R. Berman, Interaction energy of nonidentical atoms, Phys. Rev. A 91, 042127 (2015). 
[28] U. D. Jentschura and V. Debierre, Long-range tails in van der Waals interactions of excitedstate and ground-state atoms, Phys. Rev. A 95, 042506 (2017).

[29] D. P. Craig and T. Thirunamachandran, Molecular Quantum Electrodynamics (Dover, Mineola, 1998).

[30] A. Salam, Molecular Quantum Electrodynamics (Wiley, Hoboken, NJ, 2010).

[31] L. H. Ford, M. P. Hertzberg, and J. Karouby, Quantum Gravitational Force Between Polarizable Objects, Phys. Rev. Lett. 116, 151301 (2016).

[32] P. Wu, J. Hu, and H. Yu, Quantum correction to classical gravitational interaction between two polarizable objects, Phys. Lett. B 763, 40 (2016).

[33] $\mathrm{P} . \mathrm{Wu}, \mathrm{J} . \mathrm{Hu}$, and $\mathrm{H}$. Yu, Interaction between two gravitationally polarizable objects induced by thermal bath of gravitons, Phys. Rev. D 95, 104057 (2017).

[34] B. R. Holstein, Analytical on-shell calculation of low energy higher order scattering, J. Phys. G 44, 01LT01 (2017).

[35] Y. Hu, J. Hu, H. Yu, and P. Wu, Resonance interaction between two entangled gravitational polarizable objects, Eur. Phys. J. C 80, 792 (2020).

[36] H. Yu, Z. Yang, and P. Wu, Quantum interaction between two gravitationally polarizable objects in the presence of boundaries, Phys. Rev. D 97, 026008 (2018).

[37] Y. Hu, J. Hu, and H. Yu, Quantum gravitational interaction between two objects induced by external gravitational radiation fields, Phys. Rev. D 101, 066015 (2020).

[38] Y. Hu, J. Hu, and H. Yu, Induced quantum gravitational interaction between two objects with permanent quadrupoles in external gravitational fields, Phys. Rev. D 104, 086011 (2021).

[39] B. M. Axilrod and E. Teller, Interaction of the van der Waals Type Between Three Atoms, J. Chem. Phys. 11, 299 (1943).

[40] M. R. Aub and S. Zienau, Studies on the retarded interaction between neutral atoms I. Threebody London-van der Waals interaction of neutral atoms, Proc. R. Soc. Lond. A 257, 464 (1960).

[41] A. D. McLachlan, Three-body dispersion forces, Mol. Phys. 6, 423 (1963).

[42] E. A. Power and T. Thirunamachandran, The non-additive dispersion energies for N molecules: a quantum electrodynamical theory, Proc. R. Soc. London, Ser. A 401, 267 (1985).

[43] M. Cirone and R. Passante, Dressed zero-point field correlations and the non-additive threebody van der Waals potential, J. Phys. B: At. Mol. Opt. Phys. 30, 5579 (1997). 
[44] R. Passante, E. A. Power, and T. Thirunamachandran, Radiation-molecule coupling using dynamic polarizabilities: Application to many-body forces, Phys. Lett. A 249, 77 (1998).

[45] W. B. Campbell and T. A. Morgan, Maxwell form of the linear theory of gravitation, Am. J. Phys. 44, 356 (1976).

[46] A. Matte, Sur De Nouvelles Solutions Oscillatoires DesEquations De La Gravitation, Can. J. Math. 5, 1 (1953).

[47] W. B. Campbell and T. Morgan, Debye potentials for the gravitational field, Physica (Utrecht) 53, 264 (1971).

[48] P. Szekeres, Linearized gravitation theory in macroscopic media, Ann. Phys. (N.Y.) 64, 599 (1971).

[49] R. Maartens and B. A. Bassett, Gravito-electromagnetism, Classical Quantum Gravity 15, 705 (1998).

[50] M. L. Ruggiero and A. Tartaglia, Gravitomagnetic effects, Nuovo Cimento Soc. Ital. Fis. 117B, 743 (2002).

[51] J. Ramos, M. de Montigny, and F. Khanna, On a Lagrangian formulation of gravitoelectromagnetism, Gen. Relativ. Gravit. 42, 2403 (2010).

[52] H. Yu and L. H. Ford, Light-cone fluctuations in flat spacetimes with nontrivial topology, Phys. Rev. D 60, 084023 (1999). 